\title{
Complete Clinical Response in Rectal Adenocarcinoma: Review of Treatment Options
}

\author{
Brendan F Scully, C. Randall Cooper and Steven A Lee-Kong
}

Department of Surgery, Columbia University Medical Center, New York, NY 177 Fort Washington Ave, New York

Corresponding author: Brendan F Scully, M.D., Department of Surgery, Columbia University Medical Center, New York, Tel: + (201) 674-0842; Email: bfs2111@cumc.columbia.edu

Rec date: Nov 23, 2016; Acc date: Dec 16, 2016; Pub date: Dec 19, 2016

Citation: Scully BF, Cooper RC, Lee-Kong SA. Complete Clinical Response in Rectal Adenocarcinoma: Review of Treatment Options. J Clin Gastroenterol Hepatol 2016, 1: 1.

\section{Abstract}

The standard treatment for locally advanced rectal cancer is neoadjuvant chemoradiation followed by radical proctectomy with total mesorectal excision. A significant percentage of patients exhibit a pathological complete response with associated survival benefit. The management of patients who exhibit a complete clinical response after neoadjuvant chemoradiation, and could potentially avoid major resection, is a topic of great interest in colorectal surgery today. This paper reviews treatment options for this population.

Keywords: Rectal cancer; Complete clinical response; Non-operative management

\section{Introduction}

The management of rectal adenocarcinoma has evolved over the past several decades. For locally advanced rectal cancer (LARC), proctectomy is the standard of care, either through low anterior resection (LAR) for proximal and midrectal lesions, or abdomino-perineal resection (APR) with permanent colostomy for distal tumors. Surgical technique evolved from blunt dissection for negative margins to total mesorectal excision (TME), as first proposed by Heald [1]. Surgical resection is associated with significant morbidity (35\%), with approximately one third of patients experiencing urological or sexual dysfunction, or fecal incontinence [2].

A shift towards neoadjuvant chemoradiotherapy (nCRT) followed by TME has occurred, [3] with the goal of reducing local recurrence (LR) and increasing the rate of sphincter sparing procedures. After nCRT, most patients have significant downsizing of the primary tumor, and a substantial proportion ( $8 \%$ to $27 \%$ ) of these patients undergo a pathologic complete response PCR [3-5], defined as the absence of residual tumor cells in the resected surgical specimen. Patients with $\mathrm{PCR}$ have better long term outcomes, with lower LR rates and improved disease free survival (DFS) and overall survival (OS) compared to patients with residual tumor cells $[6,7]$. The rates of $p C R$ appear to increase with a longer interval between nCRT and
TME [8,9], as well as with the addition of an intensified mFOLFOX regimen prior to resection $[10,11]$. The delay in surgery does not appear to affect clinical outcomes [12,13].

While determining a $\mathrm{pCR}$ requires a complete surgical excision, there has been interest in identifying patients who exhibit a complete clinical response (cCR), determining how this correlates to a pCR, and developing different treatment strategies for these patients that avoid the morbidity of proctectomy. This paper serves to review the current literature and management options for this patient population.

\section{Complete Clinical Response}

Although pCR confers better long term outcomes, classification of $\mathrm{pCR}$ requires histo-pathological examination of a surgical specimen. A complete clinical response (cCR), defined as the absence of clinically detectable tumor after $\mathrm{nCRT}$, has been investigated as a surrogate for $\mathrm{pCR}$, with the goal of identifying patients who may be able to avoid surgery. Assessing CCR relies on a combination of physical exam, endoscopy and radiology, and determination of CCR varies among different studies. A Brazilian group, led by Habr-Gama, [14] has the longest experience with this population and has attempted to standardize the classification of cCR. An incomplete response is seen when a palpable nodule, ulceration or significant stenosis remains. Whitening of the mucosa, presence of teleangiectasia and subtle loss of rectal wall pliability are observed on endoscopy in patients with cCR. Since a transient response is common, the authors defined initial cCR occurring on first assessment $>8$ weeks after nCRT, and sustained $\mathrm{CCR}$ for patients who maintain the response from 10 weeks until at least 12 months after nCRT.

This group reported a series of 360 patients treated with $\mathrm{nCRT}$, of whom 99 patients were determined to have a CCR and were initially observed. Clinical assessment of CCR was inaccurate in 29 patients (8\%) - Five patients classified as CCR (5\%) developed early LR and underwent salvage surgery, while 24 patients $(9.1 \%)$ determined to have incomplete clinical response had pCR on histological exam [15]. The sensitivity and specificity for cCR predicting pCR in this study is $80 \%$ and $98 \%$, respectively. However, in patients with CCR who did not have LR, pCR was not confirmed with tissue sample, and clinically indolent foci of disease may remain in these patients. 
This accuracy of clinical assessment of pCR has not been replicated in other studies. Hiotis [16] reported that a CCR had a sensitivity of $77 \%$ and specificity of $16 \%$ for $p C R$. when comparing preoperative clinical exam with surgical specimen. Another study [17] demonstrated $78 \%$ accuracy in predicting pCR with a combination of endoscopy and biopsy. Other authors found that clinical examination tended to overestimate the response in patients, [18] while others found it underestimated pCR [19]. A more recent retrospective study demonstrated that most patients who demonstrate a pCR on microscopic evaluation do not display mucosal features indicative of a complete response on macroscopic examination. Clinical examination alone had a sensitivity of $26 \%$ specificity of $96 \%$ in predicting pCR [20].

These earlier studies relied mainly on clinical assessment and endoscopy, and often did not include cross sectional imaging. Improvements in radiologic studies, specifically diffusion weighted $\mathrm{MRI}$ and 18-FDG PET/CT, may improve detection of $\mathrm{pCR}$.

\section{Treatment}

For patients determined to exhibit cCR after nCRT, outside of an ongoing clinical trial, the standard of care remains surgical resection with TME. The role of non-operative management (NOM), or a watchful waiting approach, is an area of ongoing interest and research. In addition, several groups have investigated the use of local excision (LE) techniques as an alternative to TME in carefully selected patient populations or in patients considered medically unfit for radical resection. Improvements in prediction of $\mathrm{PCR}$, as well as developing an optimal surveillance regimen, the role of local excision and extended chemotherapy, need to be investigated.

\section{Non-operative management or watch-and-wait}

The Brazilian investigators from the Angelita and Joaquim Gama Institute have been the earliest and most active investigators of a non-operative approach to patients who develop a cCR. Their initial report in 2004 [21] reported outcomes of 265 patients with distal T2-T4 rectal cancer who underwent nCRT with 5-fluorouracil, leucovorin and 5040cGy radiation. At eight weeks after $\mathrm{nCRT}, 71$ patients $(27 \%)$ met criteria for $\mathrm{CCR}$ and underwent a strict watch and wait strategy that consisted of monthly examination, proctoscopy, biopsy, and CEA levels with abdominal pelvis CT scan every 6 months for the first year. After a mean follow-up of 57 months, 2 patients (2.8\%) developed endoluminal recurrence, successfully treated with either LE or brachytherapy. None developed pelvic recurrence, and 3 patients (4.2\%) developed systemic recurrence treated with systemic chemotherapy. Of the resected group, 22 patients $(8.3 \%)$ were found to have $\mathrm{pCR}$ on histological examination.

These authors updated these results in a larger patient cohort of 361 patients [22]. At eight weeks after nCRT, 122 patients (34\%) showed an initial CCR; at 1 year, 99 patients (27\%) showed a sustained cCR. Of the initial complete responders, $20 \%$ recurred within one year. For the sustained CCR, there were 13 recurrences: 5 endoluminal recurrences treated with salvage surgery, 7 systemic recurrences and 1 combined recurrence. For patients with cCR, the 5-year OS and DFS rates with $93 \%$ and $85 \%$, respectively. In 2014, Habr-Gama et al. [23] reported outcomes for all patients with an initial cCR that were enrolled in the watch-and-wait program. At, 8 weeks after nCRT, 90 patients (49\%) exhibited a cCR. With a median follow up of 60 months, 31\% developed a LR, with $60 \%$ occurring within the first year. Salvage therapy was possible for $93 \%$ of patients with a LR. Systemic recurrence occurred in $14 \%$ of initial cCR patients. For all patients with initial cCR, 5year cancer specific OS and DFS were 91\% and 68\%, respectively. Sphincter and organ preservation was possible in $86 \%$ and $78 \%$ of the cohort, respectively. When watch and wait plus salvage therapy patients were grouped together, 5 year local recurrence free survival was $94 \%$, similar to the $97 \%$ observed after TME with pCR. The authors concluded that a watch-and-wait strategy with salvage therapy may be a safe alternative for patients exhibiting a cCR after nCRT.

Some of the earlier reports come from populations that were medically inoperable or refused surgery. Lim [24] reported on 48 patients treated with $\mathrm{nCRT}$, with $56 \%$ achieving a CCR and $30 \%$ a partial response. With a median follow-up of four years, 38\% exhibited disease progression. Others reported a CCR in 10 of 58 patients who did not proceed to surgery after nCRT. Six patients suffered LR with a median time of 20 months [25].

Other groups have also investigated a non-operative approach to cCR. Maas [26] used a similar protocol to that used in Brazil, however capecitabine was used as part of nCRT regimen and high resolution $\mathrm{MRI}$ was used to screen and monitor patients. With stricter imaging criteria of $\mathrm{CCR}, 21$ patients $(11 \%)$ were followed for NOM, and compared with matched patients with $\mathrm{pCR}$ after major resection. There was one LR over a mean follow-up of 25 months, which was successfully managed with salvage therapy. Similar 2-year DFS ( $89 \%$ vs. $93 \%$ ) and OS rates (100\% vs. $91 \%$ ) were observed for CCR and $\mathrm{pCR}$ patients.

Similarly, investigators form Memorial Sloan Kettering Cancer Center [27] compared outcomes of 32 patients with CCR treated non-operatively with a cohort that was found to have $\mathrm{PCR}$ after surgical resection. Lack of visible pathology on exam and endoscopy were the primary determinants of $C C R$, and older age, lower pretreatment stage and distal tumor location were associated with NOM. With a median follow-up of 28 months, LR occurred in 6 patients (19\%) with a median time of 11 months after nCRT. All were successfully salvaged with surgery. There were no LRs in the PCR group. Two-year distant DFS and OS were similar for both groups. This experience was expanded to 73 patients with $\mathrm{CCR}$ managed non-operatively, with similar LR rates, OS and DFS rates [28].

A Danish group [29] evaluated distal T2-T3 tumors with high dose radiotherapy and oral tegafur-uracil. At 6 weeks, those with complete clinical regression, negative biopsies and no nodal or distal metastases on CT and MRI underwent a watchful waiting approach. Forty of 51 patients exhibited a 
CCR at 6 weeks (78\%), and 1-and 2-year LR rates were 15 and $26 \%$, respectively, with a median time to recurrence of 10.4 months. There were three distant recurrences. All local recurrences were salvaged with APR.

Araujo [30] compared 42 patients with cCR who underwent NOM with 69 patients with pCR after resection. Then NOM tumors were more distal, less obstructive and had a lower digital rectal score, recurrence with $28 \%$ vs. $11.5 \%$ in the NOM and PCR respectively. $80 \%$ of LR in the NOM was surgically salvaged. There was no difference in OS, but there was a significantly significant increase in DFS favoring the pCR group (61\% vs. $83 \%)$.

A recent propensity score-matched cohort analysis out of the UK [31] compared watchful waiting for 129 patients with CCR to a matched cohort who underwent surgical resection. Of the NOM group, 44 (34\%) had LR, and 36 (88\%) of 41 with non-metastatic LR were surgically salvaged. When compared with one to one matched cohorts, there was no difference in 3-year non-regrowth DFS (which excluded LR) or OS, while patients in the NOM group had significantly improved 3-year colostomy-free survival, with a $26 \%$ absolute difference in patients who avoided permanent colostomy at 3 years. This study was notable for the large number of patients and the use of MRI in determining recurrence in the NOM group.

\section{Local excision}

Local excision is an accepted surgical option for some T1 rectal cancers, but is felt to be prohibitive for tumors with greater depth of penetration due to the higher risk of nodal metastases. With the significant clinical down-staging observed with neoadjuvant therapy, several studies have explored the use of local excision after nCRT for larger tumors with the goals of achieving similar oncological outcomes without the morbidity of radical resection with TME. An alternative to TME is especially appealing to patients with very distal cancers requiring $A P R$ and patients deemed medially unfit to undergo radical resection.

A group from MD Anderson [32] published a retrospective analysis in 47patients (median follow-up 63 months) with T3 rectal cancer who underwent local excision (LE) after nCRT. Fifteen patients (32\%) demonstrated evidence of $\mathrm{CCR}$ and preferred LE, while the others refused TME (32\%), had a prohibitive comorbidity to TME (25\%) or underwent LE for undocumented reasons (11\%). The LE patients tended to be older and have smaller tumors closer to the anal verge, and a higher percentage of TME patients had N1 disease on preoperative staging than the LE group. There was no significant difference in 10-year actuarial LR, disease specific survival, DFS or OS between the LE and TME cohorts. Significantly more LE patients had no residual disease of microscopic residual disease on final pathology.

Belluco [33] reviewed the outcomes of 139 patients with T3 rectal tumors treated with $\mathrm{nCRT}$ and either TME $(n=110)$ or LE $(n=29)$. Forty-two patients (30\%) achieved pCR; these patients had significantly higher 5-year DSS and DFS rates. There was no significant difference between TME and LE in patients achieving $\mathrm{pCR}$. In the LE population, a $\mathrm{pCR}$ was significantly associated with better 5-year DFS and LR free survival. Other authors [34,35] have shown similar results of lower LR and distant metastatic rates in PCR versus no-PCR in patients treated with $\mathrm{nCRT}$ and LE.

Kundel [36] compared outcomes of patients with pCR after nCRT who had undergone radical surgery (Group 1, n=37) versus LE only (Group 2; $n=14$ ). Group 2 had a higher percentage of distal tumors and a lower post nCRT clinical stage, with 6 patients (43\%) characterized as TONO. With a median follow-up of 48 months, group 1 had four recurrences (3 LR, 1 distant), while group 2 had no recurrences. There were no significant differences in 5-year DFS, local recurrence free survival or OS between the two groups. In group 1, one patient (3\%) had evidence of nodal involvement on final pathology. The authors performed a literature review of 16 studies and found a $5 \%$ rate of positive lymph nodes (range $0 \%$ to $17 \%$ ) on patients without residual tumor on pathology after surgical resection.

There are few prospective trials investigating LE after nCRT in patients with $\mathrm{T} 1$ or $\mathrm{T} 2$ disease. Lezoche [37] reported a single institution randomized trial comparing results of TEM vs. laparoscopic TME after nCRT in T2 rectal cancer. All patients were staged as NO and were ASA class I-II. The tumors were less than $3 \mathrm{~cm}$ in diameter, within $6 \mathrm{~cm}$ of anal verge and histological grades G1-G2. Fifty patients were randomized to each group, with a median follow-up of 9.6 years and minimum follow-up 5 years. All patients achieved an R0 resection. Six patients developed recurrence in the TEM group (4 local, 2 distant) and five after TME (3 local, 2 distant). There were no significant differences in DFS (89 vs. $94 \%$ ) or OS (72 vs. $80 \%$ ) in the TEM and TME groups. The TEM group had significantly decreased operation time, blood loss, transfusions, analgesic requirements and length of stay. One patient had a suture line breakdown requiring diversion.

The multi-institutional ACOSOG Z6041 trial [38] consisted of 79 patients with T2N0 rectal cancer, less than $4 \mathrm{~cm}$ in diameter within $8 \mathrm{~cm}$ of the anal verge who received nCRT (capecitabine, oxaliplatin and radiation) followed by LE. Seventy-two patients comprised the per-protocol group with a median follow-up of 56 months. Eight (10\%) developed recurrence, 3 had LR and 5 had distant metastases. Estimated three-year DFS and OS were $88.2 \%$ and $94.8 \%$ for the intention-to-treat group, and $86.9 \%$ and $95.7 \%$ for the perprotocol group, respectively. Anorectal function and quality of life were assessed with Fecal Incontinence Severity Index (FISI) and Functional Assessment of Cancer Therapy-Colorectal (FACT-C) questionnaires at baseline and again at 12 months after treatment. There was no substantial decline in overall FISI scores or FACT-C scores at one year. Of note, due to adverse effect during chemoradiotherapy, the doses of capecitabine and boost radiation were reduced during the study for some patients.

The CARTS trial [39] investigated the use of TEM in 55 patients with T1-3N0 rectal cancer within $10 \mathrm{~cm}$ of the anal verge. Patients who achieved downsizing (ycT0-2) after nCRT with capecitabine as a sensitizer underwent TEM; patients 
whose tumors did not shrink, or had ypT2-3 after TEM, were referred for radical resection. Twenty-one patients had $\mathrm{PCR}$, while 9 had a near complete response resulting in ypT1 tumors. After a follow-up of 17 months, 4 LRs developed, one in a ypT1 patient and three in ypT2 patients who declined formal proctectomy. After nCRT, TEM enabled organ preservation in one half of the study patients. This study was notable for significant toxicity of nCRT ( 2 mortalities, 2 unable to complete) and an overall complication rate of $28 \%$ after TEM. Out of 14 complications in TEM patients, 4 required reoperations- 1 hemorrhage requiring reoperations, and 1 rectovaginal fistula and 2 presacral abscess requiring colostomy.

Pucciarelli [40] prospectively investigated LE in 63 patients with distal (defined as $<11 \mathrm{~cm}$ from anal verge) T2 or T3 rectal cancers who exhibited a major clinical response to nCRT (fluropyrimidine-based regimens). Those patients with ypTO $(n=42)$ or ypT1 with negative margins $(n=1)$ were observed, while the remaining 20 patients were referred for radical surgery, nine of whom refused. There was 1 distant recurrence and no LR in the group that was observed. There were two LR in patients with ypT2 disease, both amendable to salvage surgery. There were complications in 17 patients (27\%) after $\mathrm{LE}$; three of these patients required reoperation.

\section{Additional chemotherapy regimens}

Some groups have investigated whether adding additional systemic chemotherapy after nCRT may increase the rates of
CCR and $\mathrm{pCR}$, increasing the number of patients who may benefit from non-operative strategies.

In 2013, Habr-Gama [41] examined the long term results of patients who had CCR with an extended course of nCRT. Seventy patients with T2-4 and N0-2 rectal cancers underwent CRT with 5-fluorouracil/leucovorin and 54Gy radiation. They were assessed for tumor response at 10 weeks. In contrast to their previous studies, patients received 6 additional cycles of chemotherapy every 21 days after completion of radiation treatment. Forty-seven (68\%) patients had a cCR with 8 of these 47 developing local recurrence within the first year. Thirty-nine (56\%) patients experienced a sustained complete response for at least 12 months. Four patients developed late LR. Thirty-five (50\%) patients never underwent surgery. The rates of initial and sustained $\mathrm{CCR}$ were higher in this population with extended $\mathrm{nCRT}$ compared to the rates seen in previous studies by this group.

The addition of systemic chemotherapy and longer duration between nCRT and surgery has also been examined. In 2011, Garcia-Aguilar [11] recruited 144 patients with stage II and stage III rectal cancer. These patients were randomized to standard therapy (radiation and 5- Fluorouracil with resection at 6 weeks) or standard therapy plus 2 cycles of mFOLFOX, which delayed surgery to 11 weeks. The pCR was increased to $25 \%$ from the previously observed $18 \%$ seen at 6 weeks for the patients receiving the investigative therapy. Post-operative complications were noted to be similar between the two groups.

Table 1: Treatment options for patients with cCR

\begin{tabular}{|c|c|c|}
\hline & Key features & Areas for further investigation \\
\hline \multicolumn{3}{|l|}{ Operative } \\
\hline Radical Resection with TME & $\begin{array}{l}\text {-gold standard } \\
\text {-complete pathological staging } \\
\text {-proven low recurrence rates } \\
\text {-frequently requires ostomy }\end{array}$ & \\
\hline Local Excision & $\begin{array}{l}\text {-decreased surgical morbidity } \\
\text {-incomplete excision of perirectal lymphatic tissue } \\
\text {-avoid ostomy }\end{array}$ & $\begin{array}{l}\text {-long term outcomes } \\
\text {-post resection surveillance protocol } \\
\text {-standard surgical technique } \\
\text {-morbidity of local excision } \\
\text {-role for adjuvant therapy }\end{array}$ \\
\hline \multicolumn{3}{|l|}{ Nonoperative } \\
\hline Continued Neoadjuvant Therapy & $\begin{array}{l}\text {-avoid surgery/ostomy } \\
\text {-additional adjuvant therapy } \\
\text {-rigorous surveillance regimen } \\
\text {-salvage therapy remains an option }\end{array}$ & $\begin{array}{l}\text {-long term outcomes } \\
\text {-optimal adjuvant regimen } \\
\text {-morbidity of extended therapy } \\
\text {-optimal surveillance protocol }\end{array}$ \\
\hline Watch and Wait & $\begin{array}{l}\text {-avoid surgery/ostomy } \\
\text {-rigorous surveillance regimen } \\
\text {-salvage therapy remains an option }\end{array}$ & $\begin{array}{l}\text {-long term outcomes } \\
\text {-optimal surveillance protocol } \\
\text {-costs of extended surveillance }\end{array}$ \\
\hline
\end{tabular}

In 2015, Garcia-Aguilar [10] further examined the effect of adding mFOLFOX6 after standard nCRT in 259 patients. The control group (group 1) received CRT of $5-\mathrm{FU}$ with $50 \mathrm{~Gy}$ radiation and resection at 6-8 weeks. In groups 2-4, patients underwent standard therapy with 2, 4, or 6 cycles of mFOLFOX (leucovorin, oxaliplatin, 5-FU). $18 \%$ of control group patient 
sustained pCR with $25 \%, 30 \%$, and $38 \%$ in groups 2,3 and 4 respectively. Operative complications were similar for each of the groups with post-operative leak sand abscesses being the most common.

Duration of radiation has also come under investigation. Myerson [42] examined the effect of short course radiation on 76 patients with T3 or T4 (5 fraction in 1 week) with the addition of 4 cycles of FOLFOX followed by surgery 4-9 weeks after CRT compared to institution historic controls. Local control was noted to be $95 \%$ at 30 months, and $87 \%$ of patients had freedom from disease relapse at 40 months. These numbers were noted to be similar to historic controls.

\section{Challenges}

While recent studies suggest great potential, more investigation needs to be done before these treatment options are considered standard treatment. Most of these studies are retrospective, have small patient populations and exhibit wide heterogeneity in terms of $\mathrm{nCRT}$ regimens, surveillance protocols and length of follow-up. The definitions of cCR, nodal involvement and the distance from anal verge were not consistent among different studies, nor were the types of local excision techniques employed. In addition, the degree to which CCR correlates to PCR varies widely in the literature, and how to properly identify the patients appropriate for alternative treatments needs to improve. Recent improvements in imaging will play a major role in determining response to $\mathrm{nCRT}$.

The heterogeneity among these studies raises many questions. What is the best $\mathrm{nCRT}$ regimen? What time interval after $\mathrm{nCRT}$ should CCR is assessed? What are the best methods to determine CCR? What is the role of local excision in patients who exhibit CCR? What is the optimal surveillance protocol for patients not undergoing TME? Which patient populations may benefit from extended chemotherapy regimens? Lymph node status is the most important prognostic indicator for rectal cancer, but there is no histological evaluation of nodes in NOM. The best methods to non-invasively assess node status needs to be determined.

Another concern is the increased cost of surveillance in these alternative treatment regimens, both to the patient and the health care system. The patient undergoing NOM or LE will require frequent follow-up examinations with varying frequencies of endoscopic, laboratory and radiologic tests. This could cause significant disruption in a patient's life, and whether patients will adhere to a strict surveillance protocol outside of a clinical study is yet to be seen. Health care has become increasingly more cost conscious, and the cost of these options may make this approach prohibitive for some patients.

\section{Conclusions}

Neoadjuvant chemoradiation followed by proctectomy with TME remains the standard of care for LARC due to its low local recurrence rates and improved survival, and, outside of a clinical trial, should be offered to patients who exhibit a cCR. The associated morbidity and mortality of TME, and possibility of temporary or permanent stoma, make it an unappealing option for many patients. The observation that many patients have significant clinical downstaging after nCRT, with a significant proportion achieving a $\mathrm{pCR}$, has led to more research into rectum sparing treatment options. Table 1 lists potential treatment options for patients exhibiting a cCR.

A watchful waiting approach, with strict surveillance, seems to be a reasonable option. The current evidence shows that the majority of patients with CCR will recur within the first two years, and a multimodal surveillance approach may be able to detect LR early and enable these patients to undergo salvage surgery of either resection of additional chemotherapy. Salvage resection appears to be well tolerated with good results in many of these patients, and additional systemic chemotherapy appears to increase the PCR rates. Local excision appears to be a valid alternative to TME as salvage therapy in patients with favorable tumor characteristics.

Another approach would be LE after completion of nCRT, with patients with $\mathrm{pCR}$ referred for surveillance, while patients with residual disease referred for further chemotherapy or resection. With LE, mesorectal lymph nodes are not excised, and lymph node status is correlated with prognosis. A systemic review [43] reported that between $2 \%$ to $27 \%$ of patients with ypT0 have positive nodes on final pathology. In addition, while LE generally tolerated well with low complication rates, the addition of $\mathrm{nCRT}$ prior to LE increases complication rates in current studies.

The current management of rectal cancer involves multimodal therapy, including a combination of radiation, chemotherapy, and surgical intervention, the exact timing, duration and choice of drugs and interventions are still an area of important research. While nCRT followed by TME remains standard of care, as more research into alternatives is pursued, other treatment algorithms may become more attractive to patients and physicians. However, better prospective randomized trials are needed to validate the options presented above prior to their widespread use. There are currently several clinical trials underway (i.e. NCT02008656; NCT02052921) that we hope will improve our ability to treat rectal cancer.

\section{Conflicts of Interest}

The authors have no conflicts of interest to report.

\section{References}

1. Heald RJ, Husband EM, Ryall RD (1982) The mesorectum in rectal cancer surgery-the clue to pelvic recurrence?. $\mathrm{Br} J$ Surg 69: 613-616.

2. Schneider EB, Hyder O, Brooke BS, Efron J, Cameron JL, et al. (2012) Patient readmission and mortality after colorectal surgery for colon cancer: impact of length of stay relative to other clinical factors. J Am Coll Surg 214: 390-398. 
3. Sauer R, Becker $H$, Hohenberger W, Claus Rödel D, Wittekind C, et al. (2004) Pre-operative versus post-operative chemoradiotherapy for rectal cancer. N Engl J Med 351: 1731.

4. Janjan NA, Khoo VS, Abbruzzese J, Pazdur R, Dubrow R, et al. (1999) Tumor downstaging and sphincter preservation with preoperative chemoradiation in locally advance rectal cancer: the M.D. Anderson Cancer Center experience. Int J Radiat Oncol Biol Phycs 44: 1027-1038.

5. Pucciarelli S, Topan $\mathrm{P}$, Friso ML, Russo V, Pasetto $\mathrm{L}$, et al. (2004) Complete pathological response following preoperative chemoradiation therapy for middle to lower rectal cancer is not a prognostic factor for a better outcome. Dis Colon Rectum 47: 1798-1807.

6. Zorcolo L, Rosman AS, Restivo A, Pisano M, Nigri GR, et al. (2012) Complete pathological response after combined modality treatment for rectal cancer and long term survival: a meta-analysis. Ann Surg Onco 19: 2822-2832.

7. Maas M, Nelemans PJ, Valentine V, Das P, Rodel C, et al. (2010) Long-term outcome in patients with a pathological complete response after chemoradiation for rectal cancer: a pooled analysis of individual patient data. Lancet Oncol 11: 835-844.

8. Wolthuis AM, Pennichx F, Haustermans K, De Hertogh G, Fieuws S, et al. (2012) Impact of interval betwwen neoadjuvant chemoradiotherapy and TME for locally advanced rectal cancer on pathologic response and oncologic outcome. Ann Surg Oncol 19: 2833-2841.

9. Tulchinsky $H$, Shmuei E, Figer A, Klausner JM, Rabau M (2008) An interval >7 weeks between neoadjuvant therapy and surgery improves pathologic complete response and diseasefree survival in patients with locally advanced rectal cancer. Ann Surg Oncol 15: 2661-2667.

10. Garcia-Aguilar J, Chow OS, Smith DD, Marcet JE (2015) Effect of adding mFOLFOX6 after neoadjuvant chemoradiation in locally advanced rectal cancer: a multicentre, phase 2 trial. Lancet Oncol 16: 957-966.

11. Garcia-Aguilar J, Smith DD, Avila K, Bergsland EK, Chu P, et al. (2011) Optimal timing of surgery after chemoradiation for advanced rectal cancer: preliminary results of a multicenter, non-randomized phase II prospective trial. Ann Surg 254: 97-102.

12. Habr-Gama A, Perez RO, Proscurshim I, Santos RMND, Kiss D, et al. (2008) Interval between surgery and neoadjuvant chemoradiation therapy for distal rectal cancer: does delayed surgery have an impact on outcome? Int J Radiat Oncol Biol Phys 71: 1181-1188.

13. Kerr SF, Norton S, Glynne-Jones R (2008) Delaying surgery after neoadjuvant chemoradiotherapy for rectal cancer may reduce postoperative morbidty without compromising prognosis. Br J Surg 95: 1534-1540.

14. Habr-Gama A, Perez RO, Wynn G, Marks J, Kessler H, et al. (2010) Complete clinical response after neoadjuvant chemoradiation therapy for distal rectal cancer: characterization of clinical and endoscopic findings for standardization. Dis Colon Rectum 53: 1692-1698.

15. Habr-Gama A (2006) Assessment and management of the complete clinical response of rectal cancer to chemoradiotherapy. Colorectal Dis 8: 21-24.

16. Hiotis SP, Weber SM, Cohen AM, Minsky BD, Paty PB, et al. (2002) Assessing the predictive value of clinical response to neoadjuvant therapy for rectal cancer: an analysis of 488 patients. J Am Coll Surg 194: 131-136.

17. Kuo LJ, Chiou JF, Tai CJ, Chang CC, Kung CH, et al. (2012) Can we predict pathologic complete response before surgery for locally advanced rectal cancer treated with preoperative chemoradiation therapy? Int J Colorectal Dis 27: 613-621.

18. Benzoni E, Cerato F, Cojutti A, Milan E, Pontello D, et al. (2005) The predictive value of clinical evaluation of response to neoadjuvant chemoradiation therapy for rectal cancer. Tumori 91: 401-405.

19. Guillem JG, Chessin DB, Shia J, Moore HG, Mazumdar M, et al. (2005) Clinical examination following preoperative chemoradiation for rectal cancer is not a reliable surrogate end point. J Clin Oncol 27: 613-621.

20. Smith FM, Wiland H, Mace A, Pai RK, Kalady MF (2014) Clinical criteria underestimate complete pathological response in rectal cancer treated with neoadjuvant chemoradiotherapy. Dis Colon Rectum 57: 311-315.

21. Habr-Gama A, Perez RO, Nadalin W, Sabbaga J, Ribeiro U $J r$, et al. (2004) Operative versus non-operative treatment of stage 0 distal rectal cancer following chemoradiation therapy: long-term results. Ann Surg 240: 711-717.

22. Habr-Gama A, Perez RO, Proscurshim S, Fábio GC, Nadalin W, et al. (2006) Patterns of failure and survival for nonoperative treatment of stage $\mathrm{c} 0$ distal rectal cancer following neoadjuvant chemoradiation therapy. J Gastrointest Surg 10: 1319-1328.

23. Habr-Gama A, Gama-Rodrigues J, Sao Juliao GP, Proscurshim I, Sabbagh C, et al. (2014) Local recurrence after complete clinical response and watch and wait in rectal cancer after neoadjuvant chemoradiation: impact of salvage therapy on local disease control. Int J Radiat Oncol Biol Phys 88: 822-828.

24. Lim L, Chao M, Shapiro J, Millar JL, Kipp D, et al. (2007) Long-term outcomes of patietns with localized rectal cancer treated with chemoradiation or radiotherapy alone because of medical inoperability or patient refusal. Dis Colon Rectum 50: 2032-2040.

25. Hughes R, Harrison M, Glynne-Jones R (2010) Could a wait and see policy be justified in T3/T4 rectal cancers after chemo-radiotherapy? Acta Oncol 49: 378-381.

26. Maas M, Beets-Tan RG, Lambergts DM, Lammering G, Nelemans PJ, et al. (2011) Wait-and-see policy for clinical complete responders after chemoradiation for rectal cancer. J Clin Oncol 29: 4633-4640. 
27. Smith JD, Ruby JA, Goodman KA, Saltz LB, Guillem JG, et al. (2012) Non-operative management of rectal cancer with complete clincial response after neoadjuvant therapy. Ann Surg 256: 965-972.

28. Smith JJ, Chow OS, Eaton A, Widmar M, Nash GM, et al. (2015) Organ perservation in aptietns with rectal cancer with clinical complete response after neoadjuvant therapy. ASCO Meet Abstr 33: 509.

29. Appelt AL, Ploen J, Harling $H$, Jensen FS, Jensen LH, et al. (2015) High-dose chemoradiotherapy and watchful waiting for distal rectal cancer: A prospective observational study. Lancet Oncol 16: 919-927.

30. Araujo RO, Valadao M, Borges D, Linhares E, Ferreira CG, et al. (2015) Non-operative management of recal cancer after chemoradiation opposed to resection after complete clinical response. A comparative study. Eur J Surg Oncol 11: 1456-1463.

31. Renehan AG, Malcomson L, Emsly R, Gollins S, Maw A, et al. (2016) Watch-and-wait approach versus surgical resection after chemoradiotherapy for patients with rectal cancer (The OnCoRe project) : A propensity-score matched cohort analysis. Lancet Oncol 17: 174-183.

32. Callender GG, Das P, Rodriguez-Bigas MA, Skibber JM, Crane $\mathrm{CH}$, et al. (2010) Local excision after preoperative chemoradiation results in an equivalent outcome to total mesorectal excision in selected patients with T3 rectal cancer. Ann Surg Oncol 17: 441-447.

33. Belluco C, De Paoli A, Canzonieri V, Sigon R, Fornasarig $M$, et al. (2011) Long-term outcome of patients with coplete pathologic response adter neoadjuvant chemoradiation for CT3 rectal cancer: implications for local excision surgical strategies. Ann Surg Oncol 18: 3686-3693.

34. Guerrieri $M$, Baldarelli $M$, Organetti L, Grillo Ruggeri F, Mantello G, et al. (2008) Transanal endoscopic microsurgery for the treatment of selected patients with distal rectal cancer: 15 years' experience. Surg Endosc 22: 2030-2035.

35. Nair RM, SiegelEM, Chen DT, Fulp WJ, Yeatman TJ, et al. (2008) Long-term results of transanal excision after neoadjuvant chemoradiation for T2 and T3 adenocarcinomas of the rectum. J Gastrointest Surg 12: 1797-1805.

36. Kundel Y, Brenner R, Purim O, Peled N, Idelevich E, et al. (2010) Is local excision after complete pathological response to neoadjuvant chemoradiation for rectal cancer an acceptable treatment option? Dis Colon Rectum 53: 1624-1631.

37. Lezoche E, Baldarelli M, Lezoche G, Paganini AM, Gesuita $R$, et al. (2012) Randomized clinical trial of endoluminal locoregional resection versus laparoscopic total mesorectal excision for $\mathrm{T} 2$ rectal cancer after neoadjuvant therapy. $\mathrm{Br} \mathrm{J}$ Surg 99: 1211-1218.

38. Garcia-Aguilar L, Renfro LA, Chow OS, Shi Q, Carrero XW, et al. (2015) Organ perservation for clinical T2NO distal rectal cancer using neoadjuvant chemoradiotherapy and local excision (ACOSOG Z6041) : results of an open-label, singlearm, multi-institutional, phase 2 trial. Lancet Oncol 16: 1537-1546.

39. Verseld M, De Graaf EJ, Verhoef C, Nagtegaal ID, Punt CJ, et al. (2015) Chemoradiation therapy for rectal cancer in the distal rectum followed by organ-sparing transanal endoscopic microscurgery (CARTS study). Br J Surg 102: 853-860.

40. Pucciarelli S, De Paoli A, Guerrieri M, Mantello G, Gambacorta MA, etal. (2013) Local excision after preoperative chemoradiotherapy for rectal cancer: results of a multicenter phase II clinical trial. Dis Colon Rectum 56: 1349-1356.

41. Habr-Gama A, Sabbage J, Gama-Rodrigues J, Nadalin W, Perez RO, et al. (2013) Watch and wait approach following extended neoadjuvant chemoradiation for distal rectal cancer: are we getting closer to anal cancer management? Dis Colon Rectum 56: 1109-1117.

42. Myerson RJ, Tan B, Hunt S, Olsen J, Birnbaum E, et al. (2014) Five fractions of radiation therapy followed by 4 cycles of FOLFOX chemotherapy as preoperative treatment for rectal cancer. Int J Radiat Oncol Biol Phys 88: 829-836.

43. Martin ST, Heneghan HM, Winter DC (2012) Systematic review and meta-analysis of outcomes following pathological complete response to neoadjuvant chemotherapy for rectal cancer. Br J Surg 99: 918-928. 